Reviews in Digital Humanities

\title{
Review: Relaciones Geográficas
}

\section{Anna Lawrence ${ }^{1}$}

${ }^{1}$ University of Texas at Austin

Published on: Feb 08, 2021

License: Creative Commons Attribution 4.0 International License (CC-BY 4.0) 


\title{
Project
}

Relaciones Geográficas

Project Director

Albert Palacios, University of Texas at Austin

\section{Project URL}

https://www.arcgis.com/apps/Cascade/index.html?appid=1fcabf740a844d9d80d5bf0248416f47

\section{Project Reviewers}

Anna Lawrence, University of Texas at Austin

Keerti Arora, University of Texas at Austin

\section{Project Overview}

\begin{abstract}
Albert Palacios
The Relaciones Geográficas (Geographic Accounts) special collection site provides a simple, yet dynamic interface for researchers to explore the Relaciones Geográficas of Mexico and Guatemala, 15771585 collection at the University of Texas at Austin's Benson Latin American Collection. The collection comprises original manuscripts and maps created in response to the first survey of New Spain mandated by King Philip II, which included historical, cultural, and geographical information about the region in the 16th century.
\end{abstract}

Spanish- and English-reading students and researchers were the intended audience for this project. The site's introductory text provides the overarching historical context of the materials, comparable collections in the world, and an overview of the content and topics covered in the accounts and appended maps. The text is overlaid on some of the most stunning artifacts in the collection. At the end, an interactive point map marks politically significant towns that were extensively described in the accounts, color-coded by the Catholic Church diocese that administered the local parish. The map creator opted to manually input coordinates for each town for precision since many of the historical place names differed from the current place names. For each point, a pop-up window has been configured to provide the historical and current place name, manuscript and map creators, dates, a thumbnail, and other information about the individual items. ArcGIS Online hosts the interactive map as well as the site itself, which also provides links to the digitized maps (hosted in Omeka.net), digitized manuscript accounts (hosted in the University of Texas Libraries' collaborative transcription platform, FromThePage), raw text files for reuse in textual analysis (deposited in the Texas Data Repository), and discrete digital scholarship projects (hosted in a variety of open source platforms). 
Users can filter the towns/collection materials by the type of primary source (map and manuscript text) and the spoken Indigenous language (as indicated in the 16th-century survey response).

Anna Lamphear, Brittany Centeno, and Joey Marez, University of Texas Libraries' Digital Stewardship staff, digitized the collection materials. Albert A. Palacios, LLILAS Benson Digital Scholarship Coordinator, created the geospatial dataset, ArcGIS WebApp, and Story Map envelope, bringing in and editing contextual text that had been created for a legacy digital project. Michael Shensky, UT Libraries' GIS and Geospatial Data Coordinator, made the map's shapefile available for download/reuse through the Texas GeoData portal.

\section{Project Review}

\section{Anna Lawrence and Keerti Arora}

With the Relaciones Geográficas online exhibit, Albert Palacios has created an interactive archival tool essential for those studying the construction of colonial narratives and the spread of Spanish economic and religious impositions. The title of this project refers to a collection of responses to a 1577 questionnaire issued by King Philip II of Spain to officials in the Viceroyalties of New Spain and Peru. This order requested basic descriptive information about the geography and local culture of the settlements under Spain's rule. Forty-three of the 191 manuscript responses, termed relaciones geográficas, were acquired by University of Texas at Austin's Benson Latin American Collection from the heirs of Mexican historian Joaquín García Icazbalceta in 1937. Twenty-six of the relaciones are accompanied by hand-drawn maps, or pinturas, of the territories their manuscript depicts. Many of these were created by Indigenous artists and are useful in their own right for the study of Latin American architectural and artistic history. Since the remainder of the 167 relaciones whose locations are known are held in Seville, Madrid, and Glasgow, Benson's collection remains the only series that has been kept in the Americas. Thus, Relaciones Geográficas significantly enhances the ease of access and readability of its materials for researchers focused on 16th-century history and life.

Facilitating exploration of the collection, the project website combines a minimalist story interface with historical context and a map visualization built using the ArcGis cloud-based mapping software. The simple baselayer grayscale map of modern-day Mexican and Central American territory is populated by multicolored dots. Each represents one of the manuscripts in the collection, color-coded by the Spanish Diocese to which it pertained. Alongside the map, users are provided a widget to filter the manuscripts by such fields as "Diocese," "Manuscript Author," "Map Artist," or "Languages Spoken." By interacting with points on the map, users can access information about the modern town that the manuscript represents as well as other metadata such as the date and author of the manuscript. Each point also provides links to the item's index in the online collection, which includes 
the manuscript facsimile, its transcription and English translation, and the corresponding pintura (if available). The presentation of the interactive map as the user's initial point of engagement with this collection allows the audience to visualize the breadth of impact of the Spanish conquest in this time period and question its legacy as the spread of the reports is juxtaposed with modern and familiar Latin American borders.

Though its trajectory and potential are promising, the curation of this collection is in a nascent stage. As one clicks through the relaciones, they may discover several transcriptions and translations of the texts unavailable. While the site does not indicate a timeline for addition of these components in the collection, absent entries thoughtfully invite collaboration from readers through the FromThePage transcription platform. While the map and manuscript images are crisply rendered and technically legible, those who are unfamiliar with deciphering 16th-century Spanish calligraphy may not be able to glean much information from the facsimiles alone. Similarly, one avenue for further development is to address the accessibility of the maps themselves, both for visually-impaired researchers and for non-specialists who might need assistance in understanding the analytical and contextual aspects of the documents. The notes and questions function on the transcriptions page is rarely used but the project director might seek to partner with scholars to develop contextual materials for use in the classroom. A Colony in Crisis might serve as one model for pedagogical development of critical digital editions. Continued development of these features will fulfill the project's goals, but both Palacios and University of Texas at Austin are to be commended for investing in making these resources more widely accessible for those critically exploring Spanish colonization. 